\title{
Observation on the Curative Effect of Electroacupuncture Combined with Drugs in the Treatment of Type III Prostatitis due to Qi Stagnation and Blood Stasis
}

\author{
Wei Cao ${ }^{1}$, Qingping Zhang ${ }^{2, *}$ \\ ${ }^{1}$ Second Clinical School of Medicine, Anhui University of Chinese Medicine, \\ No. 103 Meishan Road, Hefei City (Meishan Road Campus) \\ ${ }^{2}$ School of Acupuncture and Tuina, Anhui University of Chinese Medicine. \\ No. 350, Longzihu Road, Hefei (Shaoquanhu Campus)) \\ * Corresponding Author
}

\begin{abstract}
Objective: To observe the clinical efficacy of electro-acupuncture combined with drugs in the treatment of type III prostatitis of qi stagnation and blood stasis. Methods: 70 patients with type III prostatitis of qi stagnation and blood stasis type who met the inclusion criteria were randomly divided into two groups, 35 cases in each group. The control group was given tamsulosin hydrochloride sustained-release capsules orally, and the observation group was given electroacupuncture on the basis of the control group. The course of treatment in both groups was 30 days. The National Institutes of Health Chronic Prostate Symptom Index (NIH-CPSI) score, TCM syndrome score, Self-rating Anxiety Scale (SAS) score, Self-rating Depression Scale (SDS) score before and after treatment and one month follow-up after treatment were observed level, and the clinical efficacy of the two groups was compared. Results: The total effective rates of the observation group and the control group were $91.43 \%$ and $74.29 \%$, respectively, and the difference was statistically significant $(P<0.05)$. And SDS scores were lower than those before treatment $(P<0.05)$, and the observation group was better than the control group $(P<0.05)$. Conclusion: Electroacupuncture combined with drugs has good curative effect in the treatment of type III prostatitis $(Q i$ stagnation and blood stasis type), which can improve the clinical symptoms and negative emotions of patients, and improve the quality of life.
\end{abstract}

Keywords: Type III prostatitis, Qi stagnation and blood stasis type, Electroacupuncture, Randomized controlled trial.

\section{Introduction}

Type III prostatitis (chronic prostatitis/chronic pelvic pain syndromes, CP/CPPS) is one of the common male diseases in urological outpatient clinics. Clinical manifestations[1]. At present, western medicine mainly uses antibiotics and $\alpha$-blockers to control symptoms, and the effect of single drug treatment is usually not satisfactory. The efficacy and safety of multi-drug combination treatment needs further research[2]. Acupuncture treatment of CP/CPPS has certain advantages and high safety[3]. However, at present, various treatment methods for CP/CPPS are different, and there is no unified and standardized treatment plan. From March 2021 to December 2021, the author used electroacupuncture combined with drugs to treat 70 patients with type III prostatitis of qi stagnation and blood stasis. The report is as follows.

\section{Clinical Data}

\subsection{General Data}

70 patients with type III prostatitis (Qi stagnation and blood stasis type) who were treated in the First Affiliated Hospital of Anhui University of Traditional Chinese Medicine were selected and randomly divided into two groups with 35 cases in each group. In the observation group, 23 were married and 12 were unmarried; the age was $18-47$ years, with an average of (32.85 \pm 6.78$)$ years; the course of disease was 5-30 months, with an average of $(15.80 \pm 6.69)$ months. In the control group,
20 were married and 15 were unmarried; the age was $19-48$ years, with an average of $(33.12 \pm 6.54)$ years; the course of disease was 6-35 months, with an average of (15.95 \pm 6.70$)$ months. There was no significant difference in general data between the two groups $(\mathrm{P}>0.05)$.

\subsection{Diagnostic Criteria}

(1) Western medical diagnostic criteria[4]: 1) Different degrees of lower urinary tract symptoms such as frequent urination, urgency, and incomplete urination, and no obvious abnormality in the urine routine. 2) The lower abdomen, pelvic region, and lumbosacral bulge were painful and uncomfortable, and there was no obvious abnormality in the physical examination; 3) The bacterial culture of prostatic fluid was negative.

(2) TCM diagnostic criteria[5]: Main symptoms: perineal, or external genital area, or lower abdomen, or suprapubic area, or lumbosacral, or perianal bulge, pain. Secondary symptoms: dribbling after urination, dysuria, and poor urination. Tongue pulse: dark tongue or petechiae, ecchymosis, pulse string or astringent. The main symptoms are necessary, and the secondary symptoms meet one or more, and the diagnosis can be made by referring to the tongue and pulse.

\subsection{Inclusion Criteria}

(1) Age 18-60 years old; (2) Meet the diagnostic criteria of traditional Chinese and Western medicine; (3) Have a clear consciousness, cooperate with the examination, and have 
good compliance.

\subsection{Exclusion Criteria}

(1) patients with acute urinary tract infection; (2) patients with benign prostatic hyperplasia; (3) patients with severe heart, liver, kidney and other diseases; (4) patients with prostate cancer; (5) patients who are allergic to the drugs in this study.

\section{Treatments}

\subsection{Basic Treatment}

Both groups were given health education, psychological counseling and behavioral guidance. Instruct the patient to quit drinking and avoid spicy stimulation; avoid sedentary and hold back urine; strengthen exercise; pay attention to personal hygiene and avoid unclean sexual behavior; encourage appropriate sexual behavior and avoid excessive sexual excitement.

\subsection{Control Group}

Tamsulosin hydrochloride sustained-release capsules (Astellas Pharmaceuticals (China) Co., Ltd., specification: 0.2 mg $\times 10$ capsules, approved by Chinese medicine $\mathrm{H} 20000681$ ), once a day, 1 capsule each time, with meals after oral administration.

\subsection{Observation Group}

Combined with electroacupuncture treatment on the basis of control group. (1) Point selection: RN3, RN2, LR3 (double), SP6 (double), DU20, DU26. (2) Operation method: instruct the patient to empty the urine in advance, take the supine position, routinely sterilize the operator's hands and acupuncture points, and select Huatuo brand $(0.35 \mathrm{~mm} \times 40 \mathrm{~mm}$, Suzhou Medical Supplies Factory) disposable acupuncture needles for operation. RN3 and RN4 were stabbed downward at a 45-degree angle and inserted into the needle for $35 \mathrm{~mm}$, and then the needle was lifted, inserted, twisted, and other techniques for 1 minute. RN4 is connected to a set of electro-acupuncture, SP6 and LR3 are directly punctured $30 \mathrm{~mm}$, and after deqi, the same side SP6 and LR3 are connected to a set of electro-acupuncture, continuous wave waveform is selected, the frequency is $20-50 \mathrm{~Hz}$, and DU20 is pressed downward. $20 \mathrm{~mm}$, local soreness is better, DU26 is punctured $5 \mathrm{~mm}$ upward at 45 degrees, and the needle is retained for 30min. 1 time every other day, 30min each time, treatment for 30 days.

\section{Curative Effect Observation}

\subsection{Observation Indicators}

(1) National Institutes of Health Chronic Prostate Symptom Index (NIH-CPSI): It mainly evaluates the location and degree of pain, urination symptoms, and the impact on quality of life, with a total of 43 points, and the severity is divided into mild Degree 1-14 points, moderate $15-29$ points, and severe $30-43$ points.

(2) TCM syndrome score: 6 points of swelling and pain in the main symptoms are respectively recorded as $0,2,4$, and 6 points from zero to severe; $0,1,2,3$ points; tongue and pulse 1 point; the higher the score, the more severe the symptoms, and the total score of TCM syndromes will be counted.

(3) Self-rating Anxiety Scale (SAS), Self-rating Depression Scale (SDS): Evaluate the negative emotion index of patients, of which SAS scale score of 50-59 is considered mild anxiety, 60-69 is considered moderate anxiety, $>69$ points to severe anxiety; SDS scale score 53-62 points to mild depression, $63-72$ points to moderate depression, $>73$ points to severe depression.

\subsection{Efficacy Criteria}

NIH-CPSI was used to evaluate clinical efficacy. Markedly effective: the symptoms were significantly improved, and the score was reduced by $\geq 60 \%$ compared with that before treatment; effective: the symptoms were improved, and the score was reduced by $\geq 30 \%$ compared with that before treatment; ineffective: the symptoms were not significantly improved or worsened.

\subsection{Statistical Methods}

SPSS26.0 statistical software was used for data analysis. Measurement data conforming to normal distribution were expressed as mean \pm standard deviation $(\mathrm{x} \pm \mathrm{s})$, and $\mathrm{t}$-test was used for comparison between groups; enumeration data was expressed as an example, and 2 test was used.

\subsection{Treatment Outcomes}

4.4.1 Comparison of curative effects between the two groups: see Table 1

Table 1: Comparison of curative effects between the two groups

\begin{tabular}{cccccc}
\hline Group & $\begin{array}{c}\text { Number } \\
\text { of cases }\end{array}$ & Effective & Efficient & Invalid & $\begin{array}{c}\text { Always } \\
\text { valid }\end{array}$ \\
\hline $\begin{array}{c}\text { Observation } \\
\text { group }\end{array}$ & 35 & $8(22.86)$ & $24(68.58)$ & $3(8.58)$ & $32(91.43) 1)$ \\
$\begin{array}{c}\text { Control } \\
\text { group }\end{array}$ & 35 & $4(11.43)$ & $22(62.86)$ & $9(25.71)$ & $26(74.29)$ \\
$\chi 2$ & & & & & 4.629 \\
$P$ & & & & & 0.031 \\
\hline
\end{tabular}

Note: 1) Compared with the control group, $\mathrm{P}<0.05$.

\subsubsection{Comparison Analysis}

Comparison of NIH-CPSI scores, TCM syndrome scores, SAS scores, and SDS scores before and after treatment in the two groups before and after 1 month of treatment: see Table 2 . There was no significant difference in NIH-CPSI score, TCM syndrome score, SAS score and SDS score between the two groups before treatment $(\mathrm{P}>0.05)$. The score, SAS score and SDS score decreased, and the observation group was better than the control group $(\mathrm{P}<0.05)$. 
Table 2: Comparison of NIH-CPSI score, TCM syndrome score, SAS score, and SDS score between the two groups before and after treatment $(\mathrm{x} \pm \mathrm{s})$

\begin{tabular}{|c|c|c|c|c|c|c|}
\hline Group & Number of cases & Time & NIH-CPSI score & TCM syndrome score & SAS score & SDS score \\
\hline \multirow{2}{*}{ Observation group } & \multirow{2}{*}{35} & Before treatment & $24.51 \pm 5.77$ & $17.51 \pm 4.05$ & $53.91 \pm 6.37$ & $55.48 \pm 5.97$ \\
\hline & & After treatment & $16.12 \pm 5.221) 2)$ & $12.95 \pm 3.731) 2)$ & $42.29 \pm 5.831) 2)$ & $45.51 \pm 5.981) 2)$ \\
\hline \multirow{4}{*}{ Control group } & \multirow{4}{*}{35} & Follow up & $14.26 \pm 5.381) 3)$ & $12.18 \pm 3.991) 3)$ & $38.43 \pm 5.711) 3)$ & $41.84 \pm 5.861) 3)$ \\
\hline & & Before treatment & $23.71 \pm 5.97$ & $18.31 \pm 4.11$ & $53.97 \pm 6.99$ & $54.39 \pm 5.62$ \\
\hline & & After treatment & $19.69 \pm 5.831$ & $15.53 \pm 4.271)$ & $49.31 \pm 6.641)$ & $49.62 \pm 6.031)$ \\
\hline & & Follow up & $18.03 \pm 5.521$ & $14.92 \pm 4.081)$ & $43.72 \pm 5.971)$ & $45.78 \pm 6.111)$ \\
\hline
\end{tabular}

Note: 1) Compared with the same group before treatment, $\mathrm{P}<0.05$; 2) 3) Compared with the control group, $\mathrm{P}<0.05$.

\section{Discussion}

The prostate is classified as an important part of the sperm chamber in traditional Chinese medicine, where blood and qi meet and transform sperm into a fetus. Ancient physicians have a rich understanding of the pathogenesis of CP. "The Essentials of Syndrome and Treatment: White Turbidity" states that "white turbidity is even worse... This turbid essence suffocates the resources and ends." It is believed that the turbid essence stagnating and blocking the sperm chamber is one of the main pathogenesis of CP. "Diseases": "the cells are cold and the kidney is damaged, so the urine is white and cloudy"; "Classification of Syndrome and Treatment" has a more precise understanding of the location of CP: "drenching out the urine orifice, the disease lies in the liver and spleen; turbidity out of the essence orifice, the disease lies in the heart and kidneys." Modern physicians mostly believe that diet is too obsessive, endogenous damp heat, excessive sexual intercourse, or excessive thinking, and liver qi discomfort are the pathogenic factors of CP. The bladder is unfavorable, and the patients mainly have urinary tract symptoms; in the middle stage, it is mostly damp-heat stasis intertwined, the Qi mechanism is unfavorable, the blood flow is not smooth, and the stop is blood stasis. It has been completed, the dampness and heat are not obvious, the righteousness is also unable to resist the evil and go out, the delay is difficult to heal, and the deficiency is the foundation. According to the expert consensus of the Andrology Branch of the Chinese Society of Traditional Chinese Medicine, CP is divided into syndrome types such as damp-heat injection, damp-heat stasis, liver stagnation and qi stagnation, qi stagnation and blood stasis, and kidney qi deficiency. Professor Wang Qi[6] believes that "damp heat and blood stasis block the lower Jiao" is the main pathogenic feature of CP, and "damp heat" always surrounds it. Yuan Shaoying[7] believes that "stasis" is the core pathogenesis of prostatitis, when it is classified into 6 syndromes including damp-turbid stasis, damp-heat stasis, qi stagnation and blood stasis, stasis-heat injuring yin, qi deficiency and blood stasis, and kidney deficiency and blood stasis. From the above, it can be seen that the understanding of the pathogenesis of CP by ancient and modern physicians is mostly a mixture of deficiency and excess.

RN3 and RN2 are the intersection points of the Ren and Yin meridians, which should be used for the sperm chamber in the interior, which is beneficial to the bladder and clears dampness and heat. The projection area is also located here. Through electroacupuncture, it can regulate the detrusor-urethral sphincter synergy, inhibit the activity of the TRPV1 signaling pathway, reduce the release of ATP from the bladder epithelium, and reduce the contraction frequency of ICC cells, thereby reducing smooth muscle contraction function and improving bladder compliance. The purpose of [8]. LR3, the original acupoint of the Liver Meridian of Foot Jueyin, has the functions of soothing the liver and relieving stagnation, regulating qi and blood. "Lingshu. Jianjin" points out that "the pulse of Foot Jueyin ... runs up the yin thigh, binds to the genital organs, and connects various collaterals." It can be seen that the Liver Meridian of Foot Jueyin and Qianyin are closely related physiologically; SP6, the intersection of $\mathrm{Zu}$ Sanyin, regulates the liver, spleen and kidney. Matching with Taichong can make the liver's dredging function work normally, so as to regulate the Qi machine, qi is the commander of blood, qi travels, blood travels, and when blood stasis is removed, pain is relieved. Studies have shown that acupuncture SP6 and LR3 can regulate the excitation and inhibition of the bladder and urethral nerves, thereby improving the urinary tract symptoms of patients. Symptoms of pelvic pain in patients [9-10]. Both DU20 and DU26 are points of the Du meridian. The meridian of the Du meridian connects to the brain. Acupuncture can harmonize yin and yang and flirt with emotions. The course of $\mathrm{CP}$ is long and the clinical manifestations are diverse. It has a great impact on the patient's life and work, and is very easy to repeat. With greater psychological pressure, different degrees of anxiety and depression can be seen over time. Professor Yang Jun appropriately selects the five zang-yuan points and the Rendu meridian points in the treatment of chronic diseases to adjust his spirit. On the basis of the improvement of the depressive symptoms, the patient's physical body is improved. Symptoms also recovered to varying degrees[11]. Relevant animal studies also show that acupuncture at DU20, SP6 and other points can inhibit the hippocampus by relieving the hyperfunction of the hypothalamic-pituitary-adrenal axis, increasing the expression of excitatory neurotransmitters in the hippocampus, and inhibiting the apoptosis pathway of caspase and AIF cells. Neuronal apoptosis can be reduced, and hippocampal neuronal damage can be alleviated, thereby exerting the antidepressant therapeutic effect of acupuncture[12]. The combination of various points plays the role of promoting blood circulation and removing blood stasis, clearing dampness and heat, and promoting qi and blood.

This study showed that the NIH-CPSI score, TCM syndrome score, SAS score, and SDS score of the patients were significantly improved compared with the control group after electroacupuncture combined with drugs for the treatment of qi stagnation and blood stasis type III prostatitis $(\mathrm{P}<0.05)$. The clinical efficacy of acupuncture combined with drugs is better than that of Western medicine alone, and can effectively improve the symptoms, anxiety and depression of patients, and improve the quality of life. Another disadvantage is that this study only used subjective indicators for evaluation, and other objective indicators such as magnetic resonance imaging and PSEP can be added to future research. 


\section{References}

[1] Sharp V J et al. Prostatitis: Diagnosis and treatment[J]. American Family Physician, 2010, 82(4):397-406.

[2] Mohiuddin Mohammed et al. Efficacy and safety of drug combinations for chronic pelvic pain: Protocol for a systematic review[J]. JMIR Research Protocols, 2021, 10(5): e21909-e21909.

[3] Zhou Minjie, Ying Jian, Shen Xueyong, et al. Clinical observation on the treatment of chronic prostatitis with electroacupuncture of Baihuanshu and Huiyang[J]. World Journal of Integrative Medicine, 2016, 11(01): 49-51.

[4] Wu Jieping. Urology[M]. Beijing: People's Health Publishing House, 2004: 211-234.

[5] Zhang Minjian, Bin Bin, Shang Xuejun, et al. Expert consensus on the diagnosis and treatment of chronic prostatitis with integrated traditional Chinese and Western medicine[J]. China Journal of Integrated Traditional Chinese and Western Medicine, 2015, 35(08): 933-941.

[6] Zhao Weibo, Bai Minghua, Wang Yaqi, et al. Wang Qi's experience in treating frequent urination with "main disease and main prescription", a master of traditional Chinese medicine[J]. China Journal of Traditional Chinese Medicine, 2021, 36(01): 183-185.

[7] Wang Yang, Liu Xunming. The medication rule of Yuan Shaoying in the treatment of chronic prostatitis with qi stagnation and blood stasis[J]. Henan Traditional Chinese Medicine, 2019, 39(05): 682-685.

[8] Guo Ning, Qin Hewei, Li Yanjie, et al. Based on transient receptor potential vanillic acid 1 channel to explore the mechanism of electroacupuncture in the treatment of neurogenic bladder rats after suprasacral spinal cord injury[J]. Journal of Practical Medicine 2021, 37(24): 3126-3131.

[9] Lin Chi, Zhang Lufen, Ma Liangxiao, et al. Sanyinjiao Point - characteristics of immediate analgesic effect of primary dysmenorrhea[J]. China Science: Life Science, 2016, 46(08): 1015-1022

[10] Zheng Shixu. Urodynamic evaluation of the effect of electroacupuncture at Sanyinjiao in inhibiting overactive bladder[D]. China Academy of Chinese Medical Sciences, 2015.

[11] Li Bo, Zhang Qingping, Yang Jun. Professor Yang Jun's concept of regulating the mind with acupuncture in the treatment of chronic diseases[J]. Journal of Shaanxi University of Traditional Chinese Medicine, 2018, 41(05): 146-148.

[12] Sun Yang, Tu Ya, Guo Yu, et al. Effects of acupuncture on hippocampal apoptosis-related factors in chronic restraint stress-induced depression model rats[J]. Acupuncture Research, 2019, 44(06): 412-418. 